\title{
Understanding Problem Solving in Inspiration Labs
}

\author{
Mohamed Buheji \\ Founder of International Institute of Inspiration Economy, Berrechid, Morocco \\ Email: buhejim@gmail.com
}

How to cite this paper: Buheji, M. (2017) Understanding Problem Solving in Inspiration Labs. American Journal of Industrial and Business Management, 7, 771-784. https://doi.org/10.4236/ajibm.2017.76055

Received: April 28, 2017

Accepted: June 19, 2017

Published: June 22, 2017

Copyright $\odot 2017$ by author and Scientific Research Publishing Inc. This work is licensed under the Creative Commons Attribution International License (CC BY 4.0).

http://creativecommons.org/licenses/by/4.0/

\section{(c) (i) Open Access}

\begin{abstract}
Problem solving has always been related to discipline creativity, breakthroughs, development, disruption, innovation, learning, knowledge, agility, resilience and most of all inspiration. In inspiration however, problem solving can take further meaning in that problems; complexities and challenges are sources of insights, persistence, perseverance and most of all better visualization over time. In this study we investigate the problem solving journey and see how inspiration lab contributes to its development and in tackling complex socio-economic issues. An investigation of the problem statements in the different inspiration labs carried out in the last five years is done to evaluate the importance of problem statements in creating effective solution. The paper concludes with recommendation for future research in this line to help further breakthroughs in this field where problem solving would be a source for discovering more opportunities and creating inspiration currencies that creates better social and economic results and outcomes.
\end{abstract}

\section{Keywords}

Inspiration, Inspiration Economy, Inspiration Engineering, Perseverance, Persistence, Problem Solving

\section{Introduction}

The world is full today of new challenges and unprecedented problems. These problems vary from being political, to being economical, to being social, to being technological, to being environmental and then legal.

Too many scientific, technical, complicated and specialised problem solving were proven to have limitations compared to evolving world needs and demands. Literature is in need for redefinition of the anatomy of problem solving and seeing how to create innovation and inspiration in its process [1]. The process of problem solving need to be investigated from the way it handles the 
constraints and challenges and how these can turned into possible sources for discovering opportunities.

Lately more researches have focused on tackling socio-economic problems from different perspective and ideas [2] [3] [4] [5], supporting the World Economic Forum Reports about issues on poverty, rising unemployment or income inequality, weak financial systems, gender inequality, low long term planning and investments besides last but not least the rise of non-communicable diseases (NCDs) to the prohibitive costs of care, particularly in developing countries.

There is still a gap also in the literature from the point of understanding the cognitive processes and psychological interactions that happened during the operation of problem solving which is affected by the problem statement [6] [7] [8].

In this paper the researcher would open the type of literature review that needs to be further investigated and focuses on studying the role of problem statement as one of the ways that enhance our readiness to deal with the problem anatomy, process and structure in the most suitable and possible cognitive and psychological contribution.

\section{Literature Review}

\subsection{Anatomy of Problem Solving}

What is a problem? It is a situation we want to change or an opportunity we want to discover. A well-defined problem is easy when it comes to pure science as math, physics, or even chess. i.e. you can get clear solutions, procedures and logic would play a great role in defining its outcome. The rule for the solution usually are clear, but still you have to work for it!

However in reality life is full of ill-defined problems where the rules are not clear and usually there are one "correct" solution, but there are best alternatives. Learning to solve problems is usually limited to class rooms and in formal educational settings till today. This is because mainly of not understanding the anatomy of problem solving and what it needs more. Solving a problem has never been based on instructions or discussions only, all the realised problems that were solved and helped humanity towards real development came from working on the field with trial and error.

Each problem have its constructs and codes that differentiate it from other problems. Thus each problem has its structure, specificity and complexity.

Thus each problem engages different cognitive process that needs different data collection and synthesis approach.

Thus mental activities for each problem should differ in its approach when it goes through the process of acquiring, retaining and using knowledge and it might extend even to the level when synthesis, inferences and conclusions are withdrawn.

At the time of acquiring information and knowledge about a particular problem we might go through mental image representations of either objects or events. Whether these objects or event are present or not physically present. This gradually build visualization about the problem anatomy, but with some proba- 
bility for errors [1].

\subsection{The Psychology of Problem Solving}

The competitiveness of any problem solver or problem solving methodology have always been dependent on psychology [6]. Problem solving is about thinking which in turn depends on the type and extent of the behavior directed toward attaining the solution that usually not readily available, in real life problems [9] [10] [11]. The psychology in problem solving say that we must understand the problem correctly to accurately solve it, as it is the most important cognitive activity in any daily or professional process [7].

D'Zurilla et al. [12] seen that we mostly deal cognitively with a problem the same way, as problems are rooted by our mindsets, i.e. when external event happens, we choose to see only one side of the story, and then interpret the solution around it. D'Zurilla and his team seen that we need behavioural modification as through this modification we can create a mindset that would be able to accept variety of alternative solutions. Much earlier, Norman Peale (1898-1993) have witnessed this earlier when he seen that how we think about a problem is more important than the problem itself, as how problems are stated and presented affects problem solving a great deal.

Recent studies show that inspiration physiologically is usually evoked by a problem that needs to be solved, or as a result of observing recent trends during travelling [4]. The problem is also then affected by the type of thinking [8]. For example, if our mindset is controlled by convergent thinking where our attitude would be focused on finding a particular answer to a problem, the solution would be different from that of divergent thinking where our mindest generate as many possible solutions to a problem as possible. Even the solution to a problem would if the attitude of the problem solvers tend to go for incubation thinking period where they leave the problem for a time, allowing the minds to unconsciously to find the best solution or to find from it an insight.

More research has actually shown that there are different approaches to problems solving and way of implementing innovative solutions, [13] and [14]. Found that introverts and extroverts differ even in the way brain is stimulated and how it is processed. For extroverts, their ability to take good detailed observation make their ability to tackle the problem and availability of solutions faster, however not necessarily better solutions. Therefore the introverts, might be more value added in dealing with problems that need careful monitoring and long term planning.

\subsection{Importance of Inspiring the Problem Solving Process}

The European Commission report of 2011 [15] emphasises the need for new economic resources that address the rising demands of human welfare needs, be it health care, education, resource efficiency and environmental challenges. Through inspiration, humans are self-motivated and even more equipped to solve social, economic, political, technological and environmental problems. 
Problem solving process as shown in Figure 1 start with defining the issue of the problem and therefore generating ideas from that scope. Evaluate the problem situation and see the possible ideas, then redefine the problem and make the selective decision.

In order to inspire the model of the problem solving process we need to handle the mental blocks that faces ideas and opportunity generation. The mental blocks are collection of attitudes that prevent us from thinking something different. The problem is not that there are problems, as Theodore Rubin quoted, but rather that the problem is that we are expecting otherwise and thinking that having problems is a problem. Thus we need to inspire the way we think and handle the problems before we thinking about solving them [8].

In the Handbook of Inspiration Economy we've shown how inspiration to the problem solving need to shift from the school of the few expert to the general public if we are to face the complex problems of unemployment, or to alleviated level of poverty; due to reaching sustainable limits, [4]. This means we need to shift the mindset from school of scarcity thinking to abundance thinking. Therefore one could conclude that inspiration play a role in stretching our limits as human beings thus leads to more creative solutions in solving uprising life problems.

\subsection{Stages of Inspiration of the Problem Solver}

D'Zurilla et al. [12] seen that the stages of problem solving starts with general orientation with problem and definition for its formulation, then we need to generate alternative solutions to that specific problem before we create a proper decision making.

However, in Buheji and Thomas [4] it was debated that we can't really formulate the problem characteristics sometime or even generalise it before we take it through trial and error where people would be able to try variety of solutions and eliminating those that don't work without fear.

During inspiration Lab after visualizing the problem we start dividing it into constructs and we work our way backward. The purpose of this whole process is not to solve the problem, but rather to use it build from it an insight, or find the

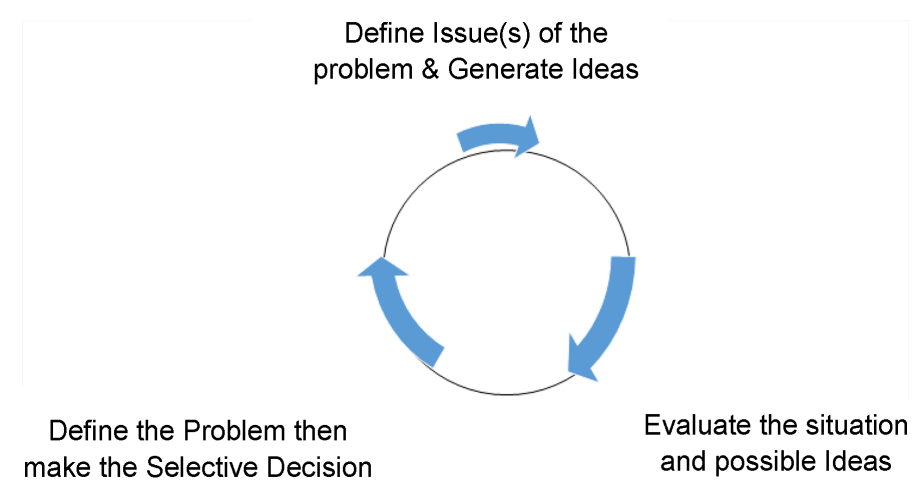

Figure 1. Problem solving process. 
blind spots that we couldn't see clearly. This move our mind from the stage of observation to stage absorption where we would be ready to realize the solution that can create real development. Therefore, one could see that insights come in literature only when the process of the problem suddenly moves from not knowing how to solve to how to solve it.

Inspiration labs that was developed as part of inspiration economy total solution not only try to solve the problem permanently, but rather looks for opportunities from inside each problem and what type of socio-economic characteristics it carries. The search for the opportunity deep inside the problem, is an inspiration currency that might not be realized at first instance. Insights or development solutions might not appear till the all the data are gathered, analyzed and deeply interpolated.

Since the solution have to meet the development goals or create a restoration we need to restate the problem before we go for identifying alternative solutions which should not be eliminated until they are tested. Then the solutions should be tested against the desired results.

\subsection{Constraints and Challenges to Inspirational Solving Problems}

In any problem solving exercise there would be always constraints relevant to time, cost and most important of all the mindset or the psychological preparedness when we are handling any problem [6].

One the most challenging psychological constraint that we tend to face when we are solving any problem till date is the functional fixedness. This is a mindset issue. The type of mental set, the inability to see an object as having a function other than its usual, or what been experienced with, build solid assumptions that leads to specific solutions. Gick and Holyaok [9] study supports this relation, since they've seen that the capacity of the brain would be able to build better analogical relations which would influence the processes of problem solving.

Constraints and challenges of the mindset define our tendencies of how we approach the problems since the habits controls our perception or thought.

\subsection{Problem Solving as a Way for Discovering Opportunities}

Our understanding of a problem depends a lot on the way we see and visaulise opportunities. The way our mindset see opportunities help us to discover hidden areas of thoughts and open for us unforeseen opportunities which are continually around us. Therefore, Sun Tzu clearly seen that real victory comes from finding opportunities in problems. This change in mindset of seeing every problem as an opportunity raise our appreciation of problems as a gift for new discovery.

The continuous handling of different problems help us to use synectics, to join two irrelevant elements of a problem or two problems to discover new opportunities. Most of the recent discoveries which comes from more than one discipline and create a disruption in product or service pathways comes from this methodology. Synectic mechanisms are very important for an inspiration based economy, since it helps to increase our probability to create an inspiration 
using our intrinsic powers. However, in order to enhance discovery of opportunities we can redesign the words, meanings, and definitions or use metaphors.

[7] and [16]. This synectic process makes the strange familiar and the familiar strange.

This methodology is heavily used in inspiration lab out which represented in Table 1. The lab works on generating lots of ideas and combining existing ideas in different ways for new purposes, seeing the same thing but thinking of something different, selecting unique and useful ideas and solutions to everyday challenges [8].

In order to keep discovering we should however avoid fixation of the mindset where we the same approach would be followed. The field work and consistent piloting in the inspiration lab helps to evaluate the alternative opportunities one at a time then to eliminate those that do not meet the desired criteria.

\subsection{Problem Solving and Quality of Observation}

One of the challenges and weak capacity for problem solving is the dependence on third party reporting where low reliability and the low quality of observation of data could be collected. Problem solving capacity differs when the information needed by problem solvers is readily available [1].

May this justify Einstein way of seeing things and where he was quoted saying that we should never impose our views on a problem; one should rather study it, till the solution reveal itself.

The locus of problem solving may iterate easier when the observation was collected by unpolluted noise, i.e. the data collected with clear use of the senses from the field, or through primary data collection that use interviews, focus group besides questionnaires and surveys. This reduce the iteration and reduce also the cost of the data collected while the availability of the information would also improve at the time needed [1]. Thus creating less complexity to the problem. Therefore Dale Carnegi used to say that the biggest problem is choosing the right thoughts, which one add that it usually comes the quality of information synthesised.

Application is a level where we ensures that the inspiration cycle is realised. Through applying the activities as codification of the problem and then classification before we do final stratification to the problem, we can make our mindset reach the status of realization. The more we collect observations through: implementation, trial and testing, changing the setting, and using different level of illustrations and demonstrations, the more we can enhance our chances to later capture and even create opportunities with confidence.

Part of the application that will help enhance our ability to discover the hidden spots inside any problem is our ability to absorb the essence of the problem and what messages it does send. In order to reach this level we need to use more classification analysis supporting with drive to enhance our forecasting. The absorption here would come more and more through modification and exploration of the problem definition and way it is presented or constructed. 
Table 1. Type of problems solved in different scopes in relevance to problem statement.

\begin{tabular}{|c|c|}
\hline Type of Business & Summary of Type of Inspiring Projects/Models \\
\hline \multirow{5}{*}{ 1) Education } & a) Shifting from Competitive Schools Focus towards Inspiring Schools \\
\hline & b) Discovering the type of inspired students vs. gifted, competitive, \\
\hline & innovative and creative students \\
\hline & c) Tracking of the inspired students after graduation. \\
\hline & d) Building Curriculums that supports students inspiration \\
\hline \multirow{10}{*}{$\begin{array}{l}\text { 2) Social } \\
\text { Development }\end{array}$} & a) Improving the Quality of Life of the Bahraini Elderly/Geriatric Care \\
\hline & Homes through inspiring their intrinsic powers ability \\
\hline & b) Inspiring the capacity of Productive Families Program to be more \\
\hline & self-independent and attractive for more family members to join as \\
\hline & employees/owners \\
\hline & c) Improving the Quality of MicroStart Families \\
\hline & d) Easing the process of home care \\
\hline & e) Supporting Working from Home Program \\
\hline & f) Revaluating the Capability of Social Allowance Entitlement \\
\hline & $\begin{array}{l}\text { g) Enhancing the products quality and competitiveness of the Retired \& } \\
\text { the Disabled }\end{array}$ \\
\hline
\end{tabular}

3) Electricity

Services

4) Police

6) Traffic

Directorate

7) Land Surveys

8) Foreign Affairs

9) Chamber of

Commerce

10) Applied

Science Colleges

11) Industry Sector

12) Commercial Sector
Improving the speed of electricity connections services 9 times faster

a) Enhancing the ability to trace Drug Trafficking and early detection

b) Enhancing the role of Prevention of Community Complains through Society Police

c) Minimising the Thefts incidents in Jewelry Shops

d) Minimisng the threat of Illegal labors

e) Minimising Families Disputes transfer to legal courts

a) Raising the speed of finishing Visa's

b) Ensuring speed \& customer services at Arrivals

a) Enhancing the appreciation of Traffic Light Violation Fines Services

b) Improving the accuracy of Traffic Accidents Investigation

Speeding up land Surveys Services

a) Ensuring the economic role of Embassies

b) Enhancement of Knowledge Sharing among Ambassadors \& Embassy Staff

Re-Establishing competitiveness for Unstable Businesses through Business Model

Inspiring students to enhance their contribution towards innovation index by more focused projects

a) Speed of throughput of Environmental friendly industrial projects that less dependent on depleting resources.

b) Enhancement of Investment utilization in the Industrial area through re-design of space utilization

a) Enhancement of CR registration through inspiring the reality of "one stop shop".

b) Improving the contribution of Microstate and Small Enterprises towards more profitability and enhancing its actual contribution to Bahraini labor Market
Type of Problem Statement

Main Technique: Basic Problem Statement

i) Observe, Clarify and Absorb

ii) Reflect on the critical blind spot

iii) Internal codification

Main Technique: Pull Thinking Stratification i) Internal Codification in relevance to types of Quality of Life Practices

ii) External Classification of potential market penetration

iii) Combination of Solutions

Main Technique: Basic Problem Statement i) Reflect on the critical blind spot

Main Technique: Pull Thinking Stratification i) Internal Codification

ii) External Classification

iii) Combination of Solutions

Main Technique: Basic Problem Statement i) Reflect on the critical blind spot

Main Technique: Basic Problem Statement

i) Observe, Clarify and Absorb

ii) Reflect on the critical blind spot

Main Technique: Basic Problem Statement i) Observe, Clarify and Absorb

ii) Reflect on the critical blind spot

Main Technique: Basic Problem Statement

i) Observe, Clarify and Absorb

ii) Reflect on the critical blind spot

Main Technique: Systematic Exploration

i) Combination of Solutions

ii) Integration of Opportunities

Main Technique: Pull Thinking Stratification i) Internal Codification

ii) External Classification

Main Technique: Pull Thinking Stratification i) Internal Codification

ii) External Classification

Main Technique: Basic Problem Statement i) Observe, Clarify and Absorb

ii) Reflect on the critical blind spot 


\section{Continued}

$\begin{array}{ll}\text { 13) Training \& } & \text { Transformation of training to make it more focused on knowledge } \\ \text { Development } & \text { management than knowledge building only in the areas of ICT and } \\ \text { Hospitality as a model }\end{array}$

14) Pension Fund Inspiring investment enhancement towards Local Market Stability

15) Water Services

16) Primary Care

17) Secondary Care

(Hospitals)

18) Secondary Care (Hospitals)

19) Public Health

20) Health

Enrichment

21) Psychiatric Services

22) Quality Assurance in

Education

23) Labor Fund

24) Municipality Services

25) Research \& Development

26) University
Minimising water loss by inspiring the ability to discover the early leakages by the process of observation

a) Early detection of Non Communicable Diseases (NCD's)

(Diabetes, Blood Pressure, Cholesterol and Obesity) by inspiring

b) Enhancement of Quality through Inspiring Families Physicians

c) Practicing Triage to inspire priority system in Healthcentres

d) Early detection of Psycho-Sematic in relevance to Anxiety in Health

Centre

Inspiring the total throughput in Accident \& Emergency and admissions in Hospitals based on Urgency of the cases

Enhancing the availability of the Capacity of Beds Utilisation by inspiring towards higher discharges on time and based on defined protocols \& followup services

Inspiration in establishing "Intelligent Inspection" that minimize the rate of poisonous food calls or low hygiene fines by $90 \%$ with less manpower resources \& trust worthiness enhancement. Thus enhancement of reputation of fast food services that supports local tourism

Enhancement of "Quality of Life" practices \& style in coordination with Health Centres

Inspiration to Manage the anxiety to avoid reaching the level of chronic anxiety where the individual would a patient treated with medicines and reduce suicide

Ensuring that level of student in under-performing school meets the minimal standard

Ensuring that all funded projects had made a success story through the domino's effect of Labor Funds

Building a comprehensive model for local people about the effect of recycling in their "Lifelong learning" abilities and "Qualities of Life" through inspiring (Schools, Families, Local Super Markets, NGO’s) to take more proactive practices toward Social Responsibility

a) Establishment of Knowledge Asset register in organization

b) Enhancement of University or the R\&D centre to deliver multi-disciplined projects

c) Enhancement of Project Closure to ensure the learning \& enhancement of projects delivery stays within the organization

d) Study the integration between the contracted projects and published papers

Ensuring Lifelong Learners Students through inspiring way of flipped class teaching and ensuring suitable preparedness for coming life challenges
Main Technique: Basic Problem Statement

i) Observe, Clarify and Absorb

ii) Reflect on the critical blind spot

Main Technique: Basic Problem Statement i) Observe, Clarify and Absorb

ii) Reflect on the critical blind spot

Main Technique: Systematic Exploration

i) Combination of Solutions

ii) Integration of Opportunities

Main Technique: Systematic Exploration

i) Combination of Solutions

ii) Integration of Opportunities

Main Technique: Systematic Exploration

i) Combination of Solutions

ii) Integration of Opportunities

Main Technique: Systematic Exploration i) Combination of Solutions

ii) Integration of Opportunities

Main Technique: New Concept Area

i) Reflect on Process of Learning

ii) Innovate \& Incubate

Main Technique: Basic Problem Statement

i) Observe, Clarify and Absorb

ii) Reflect on the critical blind spot

Main Technique: New Concept Area

i) Reflect on Process of Learning

ii) Innovate \& Incubate

Main Technique: Basic Problem Statement

i) Observe, Clarify and Absorb

ii) Reflect on the critical blind spot

Main Technique: Basic Problem Statement

i) Observe, Clarify and Absorb

ii) Reflect on the critical blind spot

Main Technique: Basic Problem Statement

i) Observe, Clarify and Absorb

ii) Reflect on the critical blind spot

Main Technique: Systematic Exploration

ii) Combination of Solutions

ii) Integration of Opportunities

iii) Start to reflect on the process of learning

Main Technique: Pull Thinking Stratification

i) Internal Codification

ii) External Classification 


\begin{tabular}{lll}
\hline 27) Labor Market & $\begin{array}{l}\text { Shifting Unemployment through inspiring the stratification of Human } \\
\text { Capital data and building models in specific industries as per countries } \\
\text { sustainable socio-economy needs }\end{array}$ & $\begin{array}{l}\text { Main Technique: Pull Thinking Stratification } \\
\text { i) Internal Codification } \\
\text { ii) External Classification }\end{array}$ \\
$\begin{array}{ll}\text { Inspiring traffic accidents reduction efforts through: } \\
\text { Traffic Accidents }\end{array}$ & $\begin{array}{l}\text { a) Enhancing the road are designed towards worst cases not best cases } \\
\text { b) High availability of road maintenance and active learning on the black } \\
\text { spots }\end{array}$ & $\begin{array}{l}\text { Main Technique: Basic Problem Statement } \\
\text { i) Observe, Clarify and Absorb } \\
\text { ii) Reflect on the critical blind spot }\end{array}$ \\
$\begin{array}{ll}\text { 29) Sanitary System } \\
\text { Enhancing drainage system design during minimisation of repeated } \\
\text { blockages in the sanitary system }\end{array}$ & $\begin{array}{l}\text { Main Technique: Basic Problem Statement } \\
\text { i) Observe, Clarify and Absorb } \\
\text { ii) Reflect on the critical blind spot }\end{array}$ \\
\hline
\end{tabular}

Once we start deep analysis of the clear absorption we might start the realisation stage. At this stage we will confidently try to arrange, connect, divide, infer, separate, classify, compare, contrast, explain, select, breakdown, correlate, discriminate the problem. At this stage we start to think empathetically, where we start to see the parts and the whole.

This should help to build synergetic practices that would lead to rational processes that involve the way of thinking and handling a problem all the way until a solution. One can argue that problem solving process and statements therefore are very important for creating better stratification (pull thinking), systematic explorations that would lead to new unforeseen concepts as shown in Figure 2.

\subsection{Human Cognitive Processes and Experiential Learning during Problem Solving}

Takashi Yamauchi [17] studied in detail the problem solving mental processes which occurs when people work toward determining the solution to a problem. Newell and Simon [18] mentioned two main approaches that move the human cognition. The first approach is called the Gestalt approach which focus on how people represent the problems. In this approach the solving of any problem involves a reorganization or representation of the problem. The other approach focus on information processing which the problem solving becomes like a search finding process.

Hippel [1] studies shown how problem solving is costly to acquire and transfer, however it is found to be very important for locus of innovation thinking and competitiveness. Training though problem solving is considered as a form of self-control and self-development program. While learning how to solve a specific problem we are actually discovering new ways of how deal with life and we are even developing our mindset to be more lean and agile. The mindset learns new attitudes, behaviors and more effective way of how to respond to challenges.

Thrash et al. [19] showed that as a result of the experiential learning that is built by trying to solve life challenging problems, as in the inspiration labs, the process of ideation occurs. 


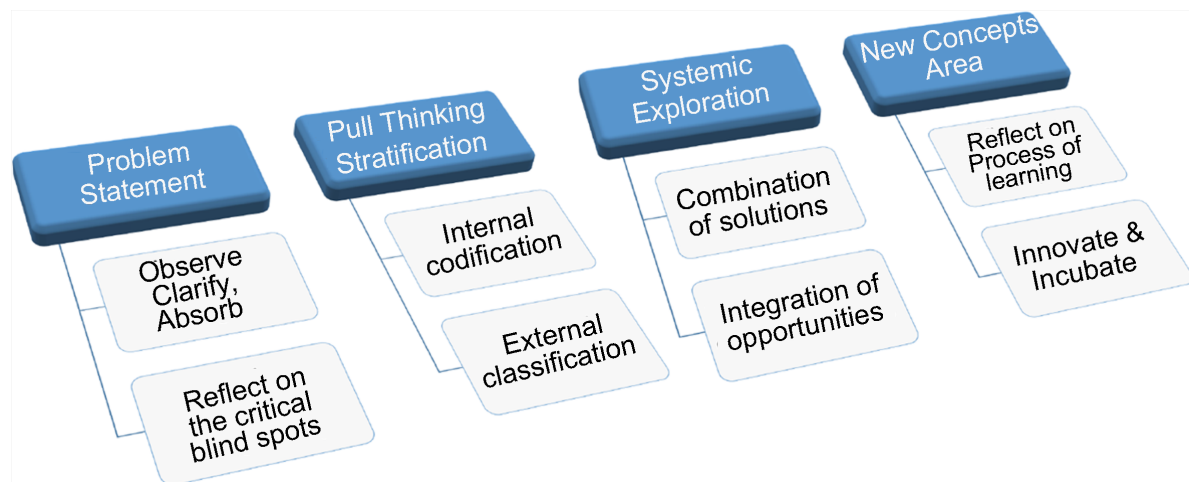

Figure 2. Use of problem statements to build up steps for concept generation.

\subsection{Distraction as a Way for Problem Solving}

Distraction is the greatest form of inspiration, because when we are distracted we are more prone to think outside of the problem. Overcoming distraction or disruption can lead to an inspiration that leads into something real and tangible. Many scientists create an intentional psychological interference with obstacles for students to stimulate their ability to learn beyond the traditional environment. [6] [10] [16].

Jack Penn said one of the secrets of life is to make stepping stones out of stumbling blocks. What prevents us from finding a solution is not distraction, but rather functional fixation. Therefore, we need to appreciate sometimes ill-structured problems since in reality they have more probability for engagement of learners.

Well-structured problems are constrained problems with convergent solutions that engage the application of a limited number of rules and principles within well-defined parameters. Ill-structured problems, even though start with fuzziness and distraction, possess multiple solutions, solution paths, fewer parameters which are less manipulable, and contain uncertainty about which concepts, rules, and principles are necessary for the solution or how they are organized and which solution is best. Jonassen [11] concluded that solving well-structured problems would be usually based on information processing that create new learning, while solving ill-structured problems cognition approaches that create a total radical change and learning.

\section{Research Methodology}

The research methodology employed in this paper is a qualitative. A longitudinal review of the experience of the 53 inspiration labs problems solved in 29 different business were set in Table 1 . These problems or challenges were encountered during the years 2012 till 2017, as part of different projects where inspiration labs problem solving techniques applied [4]. The table was set to help study the type and level of problem statements utilised to create the necessary solution to the socio-economic issues identified. The table is meant to enable the researcher to investigate the type of problem statement used for each challenge and whether it matters in creating an inspirational solutions to real-life chronic 
or challenging problems.

The paper attempts to address the research question: "What is the uniqueness of inspiration lab in identifying problem statements?" Table 1 helps also to review the scarcity of use certain level of problem statement, we the aim to investigate how we can improve the capacity for its utilization in the future. The analysis for the problem statement would be reflected through a detailed discussion that lead the conclusion.

The complexity of the type of the problems tackled shows that we can tackled lots of the world problems through inspiration labs. i.e. we can solve healthcare, poverty, educational;, unemployment, safety, environmental problems through the utilization of the process of cognitive focus that inspiration labs use and brings in.

\section{Analysis and Discussion}

\subsection{Inspiration Labs as a Tool for Generalizing Problem Solving Statement}

Inspiration Labs was introduced as a technique for complex life problem solving in early 2010 and the first paper was written about it as a reported success stories [20]. Since then lots of books and papers have been coming along in this area to cover the development and maturity of this technique over the last few years in solving problems in different field. Table 1 takes us through the unique way and variety of solutions generated from inspiration labs that were carried out by the researcher in different settings and different cultures. The design of the inspiration labs of the different issues mentioned in Table 1 helped to overcome many chronic problems that were solved through following a specific problem statement techniques. This supports the work of [7] [16] and [21]. In reference to Figure 2 problem statements found to create waves of inspiration currency in the business model of the organization and/or the targeted society. Inspiration labs shown that we can achieve new and better results, if we respond differently taking into consideration the intentions set by the level and type of problem statement as shown in the third column of Table 1 and as realized in the second column which illustrate how the problem helped us to spot better opportunities or to create new ones.

Inspiration Labs shown in Table 1 illustrates the uniqueness of problem statement in the creation of field driven ideation. This process of ideation through problem solving enhanced the level of results and even the level of organisation contribution to the socio-economy. The results of more than 190 projects, similar in complexity of those presented in Table 1 and in every discipline shows the importance of inspiration labs in raising the capacity of problem solving and in enhancing the level of knowledge integration.

The learning created by the inspiration labs problem statement has both a direct and indirect influence in creating the cycle of inspiration. The main learning of all the problem statement techniques mentioned in Figure 2 can be characterized as active, collaborative, and cooperative that leads to radical change which 
supports the work of Qin et al. [3].

\subsection{Why Inspiration Economy Needs Problem Solving Statements?}

Inspiration spirit simply can be created from a problem or a challenge or an opportunity that is built during the search for solutions. Failing to equip our mindsets with different waves of any of the problem statements as in Table 1 means we loose our focused thinking and curiosity which creates one of the main blockages of inspiration. Society that is poor in valuing and nurturing its citizen's intellectual curiosity will suffer from lack of inspiration generation abilities which in turn affects its socio-economy. This is why Inspiration Economy needs Problem Solving Statement as without it we can't maintain the minimal level of curiosity that would generate the inspiration currency [22].

Inspiration based economy needs to tackle problems in order to find opportunities for breaking the shield of the mindset. This can happen through clear problem statements. Problem statement bring in opportunities which are in unstable economy help us shift towards focusing on discovery of the intrinsic powers within the self or the targeted community.

\section{Conclusions}

Problem solving is a dynamic balance between problems solving and opportunities discovering. Through problem solving we can generate options for development. The mindset that is used to solving problems statements can have more opportunities for ideations and to identify promising ideas. Through problem solving organizations and societies we get more chances for putting our ideas into action.

Inspiration can be sustained through the ability to address appropriate problems statement. The more problems we solve, we can increase more the capacity to create judgement and to deal with failures which later build better tolerance to ambiguity.

The purpose of this paper was to explore how the problem solving statement plays a role in inspiration labs and the level of opportunities discovered. Our fundamental assumption in this paper was the relation of problem solving to the mindset. The paper shows however that the problem statement plays a role also in solving all the complex issues tackled in the inspiration labs. Thus the capacity of problem solving is not only related to the mindset, but in fact to the problem statement.

Even though this paper has limitations for finding enough previous work that goes in similar way of thinking, the researcher believes that the techniques used in categorizing the anatomy of problems and challenges faced in real life could really help in better facing more global and complex issues such the ones reported in WEF (2017) report.

This study sheds a new light for researchers about the importance of problem solving in creating better economy. It has also implications for practitioners as it 
encourages more exploration of opportunities that would inspire both social and economic aspects inside each problem by using problem statements used in inspiration labs and at different level of problem complexity.

\section{References}

[1] Hippel, E. (1994) "Sticky Information" and the Locus of Problem Solving: Implications for Innovation. Management Science, 40, 429-439.

https://doi.org/10.1287/mnsc.40.4.429

[2] Sawery, B. (1990) Concept Learning versus Problem Solving: Revisited. Journal of Chemical Education, 67, 253-54.

[3] Qin, Z., Johnson, D. and Johnson, R. (1995) Cooperative versus Competitive Efforts and Problem Solving. Review of Educational Research, 65, 129-143.

[4] Buheji, M. and Thomas, B. (2016) Handbook of Inspiration Economy. Bookboon, London.

[5] Hut, R. (2017) What Are the 10 Biggest Global Challenges? World Economic Forum 2017 Report.

https://www.weforum.org/agenda/2016/01/what-are-the-10-biggest-global-challeng es/

[6] Davidson, J. and Sternberg, R. (2003) The Psychology of Problem Solving. Cambridge University Press, Cambridge. https://doi.org/10.1017/CBO9780511615771

[7] Jonassen, D.H. (2000) Toward a Design Theory of Problem Solving. Educational Technology Research and Development, 48, 63-85.

[8] Bransford, J., Sherwood, R., Vye, N. and Rieser, J. (1986) Teaching Thinking and Problem Solving: Research Foundations. American Psychologist, 41, 1078-1089. https://doi.org/10.1037/0003-066X.41.10.1078

[9] Gick, M. and Holyoak, K. (1980) Analogical Problem Solving. Cognitive Psychology, 12, 306-355. https://doi.org/10.1016/0010-0285(80)90013-4

[10] Kendler, H.H. and Kendler, T.S. (1962) Vertical and Horizontal Processes in Problem Solving. Psychological Review, 69, 1-16. https://doi.org/10.1037/h0038537

[11] Jonassen, D.H. (1997) Instructional Design Models for Well-Structured and IIIStructured Problem-Solving Learning Outcomes. Educational Technology Research and Development, 45, 65-94. https://doi.org/10.1007/BF02299613

[12] D’Zurilla, T.J. and Goldfried, M.R. (1971) Problem Solving and Behavior Modification. Journal of Abnormal Psychology, 78, 107-126. https://doi.org/10.1037/h0031360

[13] Terwiesch, C. and Xu, Y. (2008) Innovation Contests, Open Innovation, and Multiagent Problem Solving. Management Science, 54, 1529-1543.

[14] Leonard-Barton, D. (1995) Wellsprings of Knowledge: Building and Sustaining the Sources of Innovation. University of Illinois at Urbana-Champaign's Academy for Entrepreneurial Leadership Historical Research Reference in Entrepreneurship. https://ssrn.com/abstract=1496178

[15] European Commission (2011) Innovation Union Competitiveness Report. Directorate-General for Research and Innovation, European Union, Brussels. http://ec.europa.eu/research/innovation-union/pdf/competitiveness-report/2011/iu c2011-full-report.pdf

[16] Gordon, W. (1961) Synectics: The Development of Creative Capacity. Harper \& Brothers, New York. 
[17] Yamauchi, T. (2002) The Self-Organizing Consciousness Entails Additional Intervening Subsystems. Behavioral and Brain Sciences, 25, 360. https://doi.org/10.1017/S0140525X02540061

[18] Newell, A. and Simon, H.A. (1972) Human Problem Solving. Prentice-Hall, Englewood Cliffs, NJ.

[19] Thrash, T.M., Elliot, A.J., Maruskin, L.A. and Cassidy, S.E. (2010) Inspiration and the Promotion of Well-Being: Tests of Causality and Mediation. Journal of Personality and Social Psychology, 98, 488-506. https://doi.org/10.1037/a0017906

[20] Jahrami, H. and Buheji, M. (2012) Reporting a Success Story in the Context of Public Sector: Factors That Matters. Journal of Public Administration and Governance, 2, 96-103.

[21] Jonassen, D.H. and Hernandez-Serrano, J. (2002) Case-Based Reasoning and Instructional Design: Using Stories to Support Problem Solving. Educational Technology Research and Development, 50, 65-77.

[22] Buheji, M. and Ahmed, D. (2016) In Search for Inspiration Economy Currency-A Literature Review. American Journal of Industrial and Business Management, 6, 1174-1184. http://www.scirp.org/journal/ajibm https://doi.org/10.4236/ajibm.2016.612108

Submit or recommend next manuscript to SCIRP and we will provide best service for you:

Accepting pre-submission inquiries through Email, Facebook, LinkedIn, Twitter, etc. A wide selection of journals (inclusive of 9 subjects, more than 200 journals)

Providing 24-hour high-quality service

User-friendly online submission system

Fair and swift peer-review system

Efficient typesetting and proofreading procedure

Display of the result of downloads and visits, as well as the number of cited articles

Maximum dissemination of your research work

Submit your manuscript at: http://papersubmission.scirp.org/

Or contact ajibm@scirp.org 